\author{
Marquette University \\ e-Publications@Marquette
}

Economics Faculty Research and Publications

Economics, Department of

2001

\title{
Risk Based Urban Watershed Management Under Conflicting Objectives
}

Vladimir Novotny

Northeastern University

David E. Clark

Marquette University, david.clark@marquette.edu

Robert Griffin

Marquette University, robert.griffın@marquette.edu

Douglas Booth

Marquette University

Follow this and additional works at: https://epublications.marquette.edu/econ_fac

Part of the Economics Commons

\section{Recommended Citation}

Novotny, Vladimir; Clark, David E.; Griffin, Robert; and Booth, Douglas, "Risk Based Urban Watershed Management Under Conflicting Objectives" (2001). Economics Faculty Research and Publications. 79.

https://epublications.marquette.edu/econ_fac/79 


\title{
Risk Based Urban Watershed Management Under Conflicting Objectives
}

\author{
Vladimir Novotny, David Clark, Robert J. Griffin and Douglas Booth
}

\begin{abstract}
Ecological impairment and flooding caused by urbanization can be expressed numerically by calculating the risks throughout the watershed (floodplain) and along the main stems of the streams. The risks can be evaluated in terms of the present and/or future. This article describes the methodologies for ascertaining the risks in the Geographical Information Systems (GIS) environment. The objectives of urban flood controls and ecological preservation/restoration of urban waters are often conflicting and, in the past, the sole emphasis on flood control led to destruction of habitat and deterioration of water quality.

An optimal solution to these two problems may be achieved by linking the risks to the concepts of risk communication, risk perception, and public willingness to pay for projects leading to ecological restoration and ecologically sustainable flood control. This method is appropriate because, in each case, public funds are used and the projects require approval and backing of policy makers and stakeholders. This article briefly describes a research project that attempts to resolve the conflict between the flood protection and stream ecological preservation and restoration and suggests alternative ways of expressing benefits of urban stream flood control and restoration projects.
\end{abstract}

\section{Introduction}

Urbanization has an irreversible impact on natural drainage patterns and flows in the receiving water bodies impacted by urban development. Uncontrolled development or past development in the flood plain that did not consider the impacts on hydrology, flood plain encroachment, morphology and ecology of the receiving water body system have had detrimental effects on the receiving water body, flood plain development and downstream uses of the water body. However, if development progresses in a planned, ecologically conscious way,

1 Novotny, Clark, Griffin, and Booth 
the adverse impacts on population and properties can be minimal or minimized.

Management of smaller and medium size urban streams today must today consider several objectives, such as:

\section{Controlling floods;}

2. Preserving and restoring the ecological integrity of the receiving water body affected by point and nonpoint discharges and changes in hydrology and hydraulics;

\section{Providing contact and noncontact recreation to urban populations;}

4. Optimizing other uses, such as water supply, navigation, or hydropower production. Some of the objectives conflict and some complement each other. For example, the preservation and restoration of ecological integrity and the provision of habitat for aquatic life complement recreational objectives. In fact, contact recreational uses require healthy stream ecology. On the other hand, flood control often is in conflict with ecological and recreational objectives. In the context of watershed and water body management, these conflicts must be reconciled and uses must be optimized.

\section{Hydrologic Changes by Urbanization}

Figure 1 shows a probability-frequency chart of flows in a watershed located in central Wisconsin that underwent rapid urbanization. The flows were calculated by a well-known TR-55 hydrologic runoff curve model (U.S. Soil Conservation Service) after entering the watershed characteristics as they are changed by urbanization. The watershed area is $36.7 \mathrm{~km}^{2}(14.2$ sq.mi.). The original use of land in the watershed before 1960 was as a rural mix of agricultural and forested lands. By the time of the 1985 Federal Emergency Management Administration (FEMA) flood delineation study, the watershed became about 20 to 25 percent urbanized. Soils of the watersheds were predominantly in the U.S. SCS hydrologic categories of B and C (loams and silt loams). As of 1998, the watershed was about 40 percent urbanized and the dominant land use had become residential. The other current, major land uses are transportation (including a freeway and state and local highways) and a large shopping mall. As Figure 1 illustrates, the flow that was a 100-year flood in the 1965 pre-development period is today a high flow occurring on average every three to four years and could become an annual high flow when the watershed is fully developed.

The increased magnitude and frequency of high flows have several major adverse effects on the community located near the water course, on the floodplain, and on the ecology of the

2 Novotny, Clark, Griffin, and Booth 
urban stream. Hydrologic effects can be summarized as follows:

1. The floodplain enlarges. In the United States, the floodplain is defined as an area that is flooded up to the extent of the 100 year flood. Figure 1 shows that as the magnitude of the 100 year flood increases, areas that were outside the 100 year flood plain would become a part of it.

\section{The frequency of flooding inside the floodplain increases. Under natural} conditions, a river channel overtops about once every 1 1/2 to two years (Leopold, Wolman and Miller, 1992). Figure 1 documents that the channel, as a result of urbanization, is overtopped several times each year.

3. Peak flows during storm events are increased. Since surface flow moves faster, the time of concentration is decreased.

\section{The magnitude and frequency of all runoff events of all sizes increases. This} outcome is especially important for rainfalls of smaller and medium magnitudes. Before urbanization these smaller rainfalls mostly infiltrated into soil and the flows in the stream were smaller and could be easily contained in the natural channels of the stream. After urbanization the same medium rainfall could result in a flood.

5. Channels become unstable and more erosive (degrading) as a result of increased medium floods (Booth and Jackson, 1997). This outcome has an adverse impact on habitat.

\section{Imperviousness of the watershed impedes recharge of shallow groundwater} aquifers. This outcome diminishes the base flow contributions. Some streams may become ephemeral or effluent dominated.

7. More flow moves on the surface, and with a faster velocity. This outcome increases the volume of surface runoff contribution.

Urban engineers in the past had tried to resolve the problem of increased floods by enlarging the flow capacity of urban streams via the processes of lining, covering, and straightening the channels. Los Angeles River in California and the Kinnikinnic River in Milwaukee are examples of the ultimate transformation of urban streams into concrete high flood velocity channels with very little biological habitat. At the same time, flood-plains were being lost to urban development. In general, most urban stream modification projects are driven by the

3 Novotny, Clark, Griffin, and Booth 
need to control floods. However, economics are not favorable to projects that consider only flood control needs and most urban flood control projects have negative net benefits, i.e., the project costs far exceed the benefits in terms of flood damage reduction. This article briefly describes a research project that

(1) makes an attempt to resolve the conflict between flood protection goals and stream ecological preservation and restoration needs, and (2) explores alternative ways of expressing the benefits of urban stream flood control and restoration projects.

\section{Risks of Floods and Ecological Integrity}

In order to compare the risks of increased flooding and the deteriorated ecological integrity of urban streams, the first step is to define a measure for both. Ideally, these measures should be comparable. But that, at this point, seems impossible. The next best solution is to assign weights so they can be compared.

\section{Measure of Flood Risk}

There is a need to express a flood risk relation in the urban flood plain. First, let us define a flood as a flow that is greater than the capacity flow of the channel. A floodplain is a part of the river corridor (Figure 2).

It is also necessary to expand the probabilistic definition of flooding to areas away from the channel. As one moves away from the river's edge (the beginning of the flood plain) the probability of flooding decreases and at some point at a distance $X$ from the river's edge the recurrence interval of flooding becomes 100 years, i.e., the risk of flooding is $r(X)=\mathbf{0 . 0 1}$. This is the extent of the 100 year flood plain that defined and delineated for engineering and flood insurance purposes. The schematic of the risk is then shown on Figure 2. If before urbanization the smallest flow that leaves the channel is about a flow with a recurrence interval of two years (Figure 2) then the annual risk of flooding at the bank of the river is $r_{n}(\mathbf{0})=1 / 2=\mathbf{0 . 5}$. If, as a result of urbanization, flooding becomes more frequent, for example, if the bankfull capacity flow is exceeded twice a year, the risk of flooding at the river's bank becomes $r_{u}(0)=1 / 0.5=2$ and so forth. The subscripts $\boldsymbol{n}$ and $\boldsymbol{u}$ denote natural (pre-development) and urbanized (post development) conditions, respectively. The monthly probability (risk) can be calculated from a series of maximum monthly flows and not just from one, per-year maximum flow. This approach enables us to consider the fact that there may be more than one occasion in a year when the

4 Novotny, Clark, Griffin, and Booth 
flow leaves the confines of the channel and becomes a flood. Thus, the monthly risk will be slightly different from $1 / 12$ of the annual risk that is based on only one flood per year. To bring the magnitude of the risk on par with the water quality risks that are expressed in terms of the probability of daily grab or four day composite samples exceeding the acute or chronic toxic concentrations or water quality criteria, the risk of monthly flood would be further divided by 30.41 .

The logarithmic mathematical form of the risk function is selected for convenience and simply expresses the fact that floods on rare occasions may extend further than the 100 year flood plain limits. The risk function can be then expressed as $r=C_{10}{ }^{-K x}$.

The function parameters can be easily estimated from the knowledge of the risk of exceeding the bankfull capacity flow and from the extent of the 100 year flood plain. $C$ in the above equation is the risk of exceeding the bankfull flow, or, $\mathbf{C}=\mathbf{r}(\mathbf{0})$. In the Geographical Information Systems (GIS) environment, the risk function can be ascertained from flood flow elevations and contours of the flood plain.

This risk function can be integrated, i.e. see Figure 3, where subscripts $I$ and $\boldsymbol{r}$ correspond to left and right bank flood plains. The flood plain risk parameter, $\mathbf{R}$, or function, $\mathbf{r}$, can be combined with the flood damage cost information to yield annualized flood damage indicator. If $\mathbf{d}$ is a uniform flood damage cost expressed in dollars $(\$)$ per $\mathrm{m}^{2}$ of the flood plain then the total annualized flood damage function is simply $\mathbf{D}=\mathbf{R} \mathbf{x} \mathbf{d}$. The unit of the annualized flood damage indicator is $\$$ (meter of length of the stream) ${ }^{-1}(\text { year })^{-1}$. If $\mathbf{d}$ varies with the distance from the stream then this function is included into the integral (Equation 2) and solved. This parameter could be used in flood risk communication. The flood damage cost, $\mathbf{d}$, includes a variety of the costs of remedies for flooding, such as pumping water from basements and streets, cleaning up flooded basements, repair of houses damaged by flooding, cleaning roads, loss of property, and loss of time. It represents the total cost of cleanup and repair divided by the flooded area.

The concept of flood risks can be incorporated into a GIS environment. The information and data necessary to develop the flood risk are obtainable from flood insurance maps, U.S. Geological Survey stage-flow rating curves, watershed elevation contours, and conventional hydrologic and hydraulic engineering calculations.

5 Novotny, Clark, Griffin, and Booth 


\section{Ecological Risk}

Following U.S. Environmental Protection Agency (EPA) (1992) and WERF (Parkhurst et al, 1996) risk assessment documents, ecological risk for aquatic systems is defined as "a probability that a genus residing in or potentially indigenous to the receiving water body will be lost or acutely damaged by existing or potential discharges of pollutants." The term potentially indigenous reflects the fact that the representative composition of organisms should be selected from a composition in similar unimpacted water bodies located in the same ecoregions.

The calculations of individual risks for each stressor are demonstrated in Figure 3. EPA currently evaluates ecological risks in terms of the loss of species or genera that will result from the environmental impact (Parkhurst et al., 1996, US EPA, 1992). This risk is basically a joint probability function of (1) probability density function of concentrations, $\mathbf{f ( E M C )}$, and (2) probability that species will be lethally or chronically impacted when exposed to a given concentration, $\mathbf{g}$ (R EMC). A simple model and method for calculating ecological risks of contaminants present in storm-water discharges was published by Novotny and Witte (1997). The method assumes that the event mean concentrations of pollutants are log-normally distributed. At this point the method estimates only the risk of acute damage to the indigenous population. Both storm-water and base flow discharges are considered. The method considers dilution of storm-water and CSO discharges and Water Effect Ratio. A simple soft-ware package has been developed by the Water Environment Research Foundation (Parkhurst et al., 1996). The single, dimensionless risk value has numerous advantages over the traditional separate comparison of measured water quality data with criteria because it puts all pollutants on the same basis, i.e., the probability of ecological damage to the resident biota (or potentially resident as derived from reference unimpacted water bodies of the same character within the ecoregion). It may also be an additive and comparative number, i.e., risks from several compounds and those from dry weather discharges could be added together to yield an overall risk and approximate synergy and individual risks can be quantitatively compared.

\section{Willingness to Pay - A Weighting Factor for Risks}

As shown above, the ecological and urban flooding risk can be expressed in apparently similar units; however, these risks are not directly comparable and need not be valued the same by local residents. A solution is to assign weights to each risk and use a common denominator to develop the weights. One technique that has been used by economists to assess the underlying

6 Novotny, Clark, Griffin, and Booth 
value associated with nonmarket goods such as environmental and flooding risk reduction is the contingent valuation method (CVM). This approach employs a survey of residents to assess their maximum willingness to pay (WTP) to improve, or avoid degradation of a local resource. In the current context, the approach can be used to determine WTP for reduced ecological risk, and the maximum WTP to avoid increasing flooding risks above existing levels as a consequence of continued urbanization. The stated WTP payment should be that dollar value that would make the respondent indifferent between the original level of risk and the hypothesized change in risk. Hence, the WTP response gives an indication of the true value to the respondent of the hypothetical change being described in the questionnaire. If carefully designed, these surveys can be used to not only gauge the level of public support for a project, but also determine the community benefits associated with public investments in flood control and ecological improvement to urban watersheds.

This technique is being employed in the present analysis of flooding and environmental risks in two watersheds within the Milwaukee metropolitan area. Development scenarios are described to the respondents based on ecological and hydrological simulation. Respondents are then asked to define maximum annual payments to (1) prevent increases in flood risks associated with development scenarios and, (2) improve ecological quality of the river and its environs. Although the economic theory that underlies the CVM has been thoroughly developed by Carson and Mitchell (1989), and others, there are a number of issues outlined below that are relevant to this particular application.

\section{Descriptions of Public Goods}

The respondents must understand clearly what is being valued and, equally important, the respondent must understand what is not being valued. For example, in the Milwaukee area, there have been consistent problems with sewer backups and basement flooding due to inadequate grading around houses. We are careful to point out that the flooding risks being valued are related to the rivers and streams overtopping their banks, rather than these alternative forms. To assist in the development of accurate and believable descriptions of the flood risk and ecological risk goods, focus groups drawn from residents within the watershed were conducted.

7 Novotny, Clark, Griffin, and Booth 


\section{Referendum Format, Fiscal Reference Points and the Payment Vehicle}

Since most flood control and environmental improvement projects are financed publicly, we used a referendum format to describe the project. That is, the project was described to the respondent in the form of a public referendum that would be financed with public tax dollars. Residents were then given as reference points average public expenditures on numerous public services provided by their state and local government (e.g., annual expenditures on police, fire, education, ambulance, etc.). We were careful to avoid identifying the property tax as the specific payment vehicle for the referendum. Rather, it was indicated that the payment would be made through a combination of state and local taxes. Given that most local flood control projects are financed over a period of 20-30 years, we indicated a 20 year period for the financing of the project. The respondent was presented with a randomly generated project cost between $\$ 0$ and $\$ 500$ (the range was determined from the focus groups) and asked if they would be willing to vote for the project if it were on the next ballot. This permits the determination of median responses to the WTP question. Furthermore, respondents were also asked to state the most they would be willing to pay in annual tax contributions over the next 20 years.

\section{The Embedding Issue}

A debate has developed as to whether WTP responses truly reflect the valuation of the good in question, or whether they simply reflect a desire to purchase moral satisfaction that one is actually doing something about an environmental problem (embedding). To test whether embedding is a problem among our respondents, we ask different WTP questions for three distinct groups of respondents. The first set of respondents was asked to place a value on maintaining the status quo on flood control in light of continued urbanization. A second group was asked to value ecological improvements to the watershed, and the third group was asked to value a project that included both ecological risk reduction and flood control. If embedding is not a problem, then the average WTP of the third group would be greater than that of either the first or the second groups. If completely separable, the WTP of the first two groups would sum to that of the third group.

\section{Studying Watershed Residents}

A two-wave, panel-design, probability sample survey of more than 1000 adult heads of households residing in two pilot watersheds (Oak Creek and the Menomonee River) located in

8 Novotny, Clark, Griffin, and Booth 
the Milwaukee Metropolitan area is being conducted by telephone in 1999-2000 to ascertain WTP for flood control and/or ecological restoration of these two degraded watersheds. The interviews also ask most respondents questions that examine carefully various potential predictors of WTP, including a series of sociodemographic, attitudinal, risk perception, and risk communication variables based on a model of risk information seeking and processing developed by Griffin, Dunwoody and Neuwirth (1999) from powerful psychological theories such as the Theory of Planned Behavior (1988) and the Heuristic-Systematic Model of information processing (Eagly and Chaiken, 1993). The panel design of the study (i.e., reinterviewing the same individuals over time, with some new individuals added in the follow-up interview to control for sensitization effects) allows us to assess patterns of likely influence among the variables as well as the stability or volatility of WTP estimates over time.

Prior to the start of the survey, eight focus groups, consisting of citizens recruited in the watersheds, had been conducted in Spring 1999 to help develop survey questionnaire measures, including finding the best lay terminology for posing the WTP questions and describing flooding and ecological risks to survey interviewees. Because focus groups, even those drawn from samples, tend to be unrepresentative of the larger population, results were not used directly for policy or planning guidance

\section{Theory of Planned Behavior}

The Theory of Planned Behavior (Ajzen, 1988), which has been successfully tested in prediction of a wide range of human behaviors, is being applied in this study as a means of determining some key predictors of WTP. In this study, WTP is considered to be a form of Behavioral Intention (here, an intention to pay a particular amount for the described benefit in terms of flood risks or ecological improvements). Ajzen's theory indicates that behavioral intention $(\mathrm{BI})$ is predicted by a limited set of psychological variables, notably one's sense of control over the behavior (e.g., the amount one could pay), one's social normative beliefs (e.g., one's sense that other people important to the individual would want him or her to pay for the benefit), a set of beliefs about the cost-benefit consequences of performing the behavior (e.g., that paying a given amount would in fact help people who live in the flood plain), and a set of values that the individual holds about those consequences (e.g., that helping people who live in the flood plain in this way is a desirable outcome). The latter two elements (outcome beliefs and outcome evaluations) are considered to be the building blocks of an attitude toward performing 
the behavior, which is the more direct predictor of $\mathrm{Bl}$. The theory should serve as a diagnostic tool (e.g., what separates those people who are willing to pay for flood control or environmental improvement from those who are not?) as well as a predictor.

\section{Risk Communication and Perception}

One dimension of WTP of key interest to researchers and policy makers is the stability (or volatility) of public WTP over time. Volatile WTP estimates mean that policy makers cannot truly plan on the level of public support voiced in WTP surveys. Thus this study seeks to determine factors that lead to stability in WTP estimates. In general, factors that contribute to stability in the variables that Ajzen (1988) uses as predictors of BI should contribute indirectly to stability in $\mathrm{BI}$ (WTP).

To examine these factors, we apply the model of risk information seeking and processing (Griffin et al., 1999). The model proposes that seven factors -- (1) individual characteristics, (2) perceived risk characteristics, (3) affective responses to the risk, (4) felt social pressures to possess relevant information, (5) information sufficiency, (6) one's personal capacity to learn, and (7) beliefs about the usefulness of information in various channels -- will affect the extent to which a person will seek information about the risk in both routine and nonroutine channels and the extent to which he/she will spend time and effort analyzing the risk information critically (i.e., "processing" it). A key aspect of this model is its reliance on Eagly and Chaiken's (1993) Heuristic-Systematic model, which proposes that information that is analyzed more systematically will produce attitudes more resistant to change over time. Thus, we would expect that factors that lead people to spend more effort gathering and analyzing information about flood risks and about risks to the urban watershed ecosystem will develop more stable beliefs and attitudes toward paying for flood control and ecosystem improvement and, therefore, will provide more stable WTP estimates.

Environmental values are a special form of beliefs about how things should be in the world and about what we should do to make the world a better place. For instance, a basic environmental value could be a belief that restoration of urban watersheds is the right thing to do. Environmental values can be anthropocentric (i.e. human centered) or they can be biocentric (Norton 1995). In the case of anthropocentric values, environmental improvement should be undertaken only for the benefit of people. For biocentric environmental values, ecological improvement should be undertaken both for the benefits humans and for the sake of nature itself.

10 Novotny, Clark, Griffin, and Booth 
The surveys were designed to find these attitudes.

\section{Synthesis}

Using the model of risk information seeking and processing (Griffin et al., 1999) and the Theory of Planned Behavior (Ajzen, 1988) enables us to predict variance in WTP and helps us explain cognitive, attitudinal, and social normative reasons for WTP. The WTP behavioral intention of respondents, as well as its cognitive and attitudinal precursors, are investigated by considering numerous factors such as upstream (source) vs. downstream (impact) location, living or owning real estate inside or outside the floodplain, other demographic parameters including the standard of living, and finally, the measured and calculated flooding and ecological risks. The estimated WTP functions can then be evaluated across various policy simulations to make derive the benefits associated with risk reductions. These benefit estimates can be compared with project costs to evaluate the economic efficiency of proposed projects.

\section{Conclusion}

This project represents an interdisciplinary analysis of two degraded urban watersheds. Hydrologic and biological models have been developed to quantify the impact of urbanization on flooding and ecological risks in two urban watersheds. The social scientists on the research team then derive individual and public values for such risk reductions. Although much of the engineering and biological work has been completed, the first wave of the survey was completed in April 2000, and the second wave will follow in the Fall and Winter of 2000/2001. Once the analyses of these surveys are completed, policymakers and stakeholders will be able to address a number of important issues. These include:

1. What are the relative weights placed on WTP for flood control vis a vis risk reductions for environmental quality in urban rivers, and how do these vary with individual and neighborhood circumstances?

2. What role does communication and public education play in determining WTP?

3. What factors lead to political support for flood control and ecological risk reductions?

4. Under what circumstances can officials count on continued public support in monetary terms (i.e., stability of WTP estimates over time)?

5. In light of future urbanization trends, how do the derived benefits associated with flood

11 Novotny, Clark, Griffin, and Booth 
control and watershed ecological improvement projects compare with the costs of these projects?

Because the location and other descriptors of respondents to the survey are known, the results will also enable the researchers to assign approximate spatial or neighborhood weights to these two types of risk, thereby reflecting community support for flood control and ecological risks. This outcome will help stakeholders to assign funding priorities. Preliminary results of the focus groups and of the first survey indicate that the WTP estimates in pilot watersheds are generally greater than the cost of the remediation, especially when ecological restoration and preservation are considered. Preliminary results of the survey indicate that 78.6 percent of respondents either strongly agree or agree with the statement that "the health of urban rivers should be improved for the sake of nature itself'. This clearly suggests that the public in Milwaukee urban watersheds subscribe to biocentric urban values.

\section{Acknowledgment}

The research described in this presentation is sponsored by the Research Grant No. EPA Grant Number: R82-5759 from the US Environmental Protection Agency as a part of the EPA/NSF/USDA STAR Watershed Program. The views expressed in this presentation are those of the authors and not of the sponsoring agencies.

12 Novotny, Clark, Griffin, and Booth 


\section{References}

Ajzen, I. (1988) Attitudes, Personality and Behavior. Open Univ. Press, Milton Keynes, UK.

Booth, D.B and C. R. Jackson (1997) "Urbanization of aquatic systems: Degradation thresholds, stormwater detection, and the limits of mitigation," Journal of AWRA, 33(5):1077-1090.

Carson, R.T., and R.C. Mitchell (1989) Using Surveys to Value Public Goods: The Contingent Valuation Method, Resources for the Future, Washington, DC.

Eagly, A.H., and S. Chaiken (1993) The Psychology of Attitudes. Harcourt Brace, San Diego.

Fishbein, M. and I. Ajzen (1975) Belief, Attitude, Intention, and Behavior: An Introduction to Theory and Research. Adison-Wesley, Reading, MA.

Griffin, R.J., S. Dunwoody and K. Neuwirth (1999) "Proposed model of the relationship of risk information seeking and processing to the development of preventive behaviors," Environmental Research, Section A, 80:S230-245.

Leopold, L.B., M. G. Wolman, and J.P. Miller (1992) Fluvial Processes in Geomorphology. Dover Publications, New York.

Norton, B.G. (1995) "Why I am not a nonanthropocentrtist: Callicott and the failure of monistic inherentism," Environmental Ethics 17:341-358.

Novotny, V. and J.W. Witte (1997) "Ascertaining aquatic ecological risks or urban stormwater discharges," Water Res. 31(10):2573-2585.

Parkhurst, B.R. et al. (1996) Aquatic Ecological Risk Assessment: A Multi-tiered Approach. Project 91-AER-1, Water Env. Research Foundation, Alexandria, VA.

Plafkin, J.L., M.T. Barbour, K.D. Porter, S.K. Gross and R.M. Hughers (1989) Rapid Bioassessment Protocols. EPA 444/4-89-001, U.S. Environmental Protection Agency, Washington, D.C.

U.S. Environmental Protection Agency (1992). Framework for Ecological Risk Assessment. Risk Assessment Forum. EPA/630/R-92/001, Washington, D.C.

13 Novotny, Clark, Griffin, and Booth 


\section{Appendix}

Figure 1: Effect of Urbanization on Flood Flows in a Small Midwestern (US) Watershed

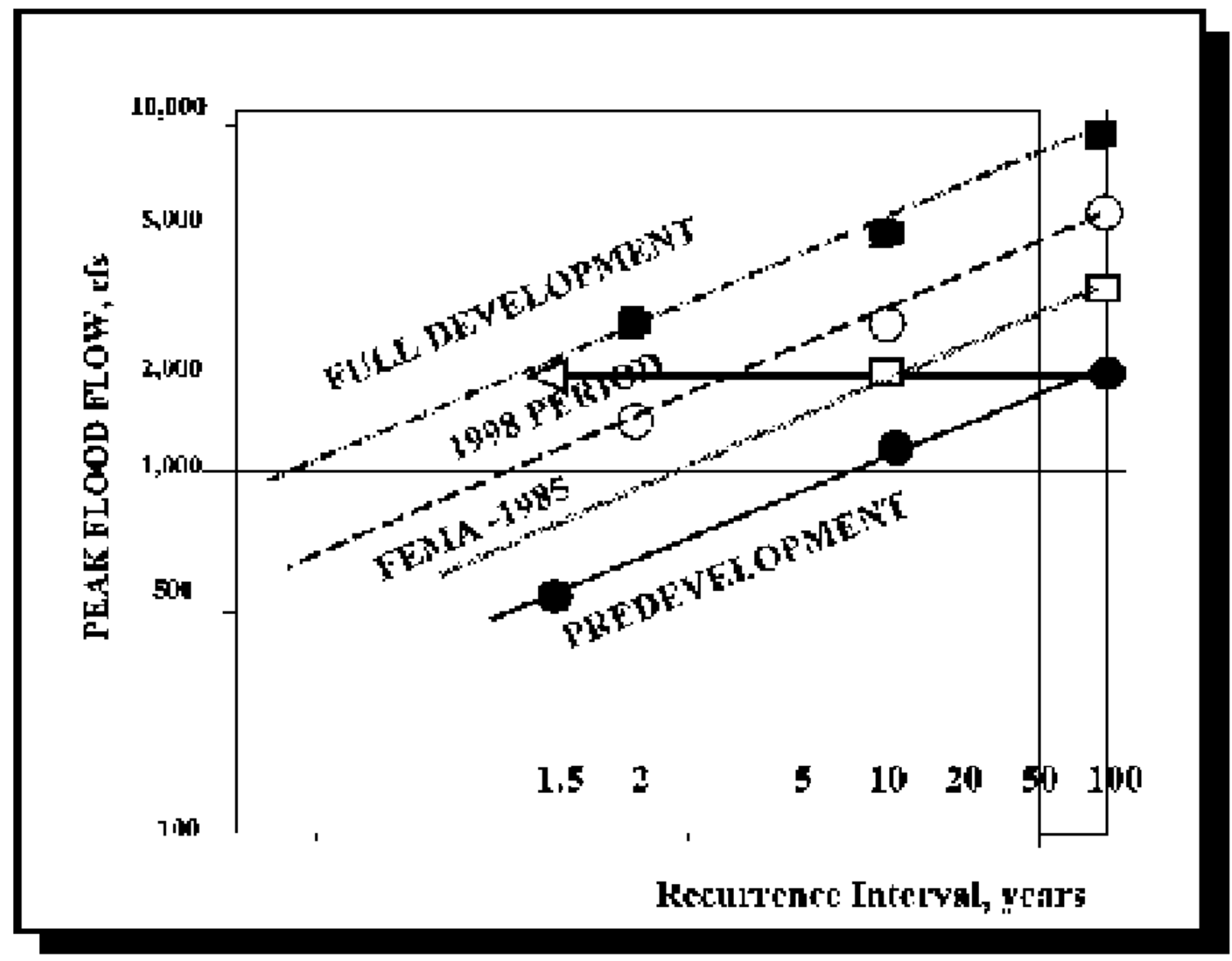

14 Novotny, Clark, Griffin, and Booth 
Figure 2: Concept of Urban Flood Risk

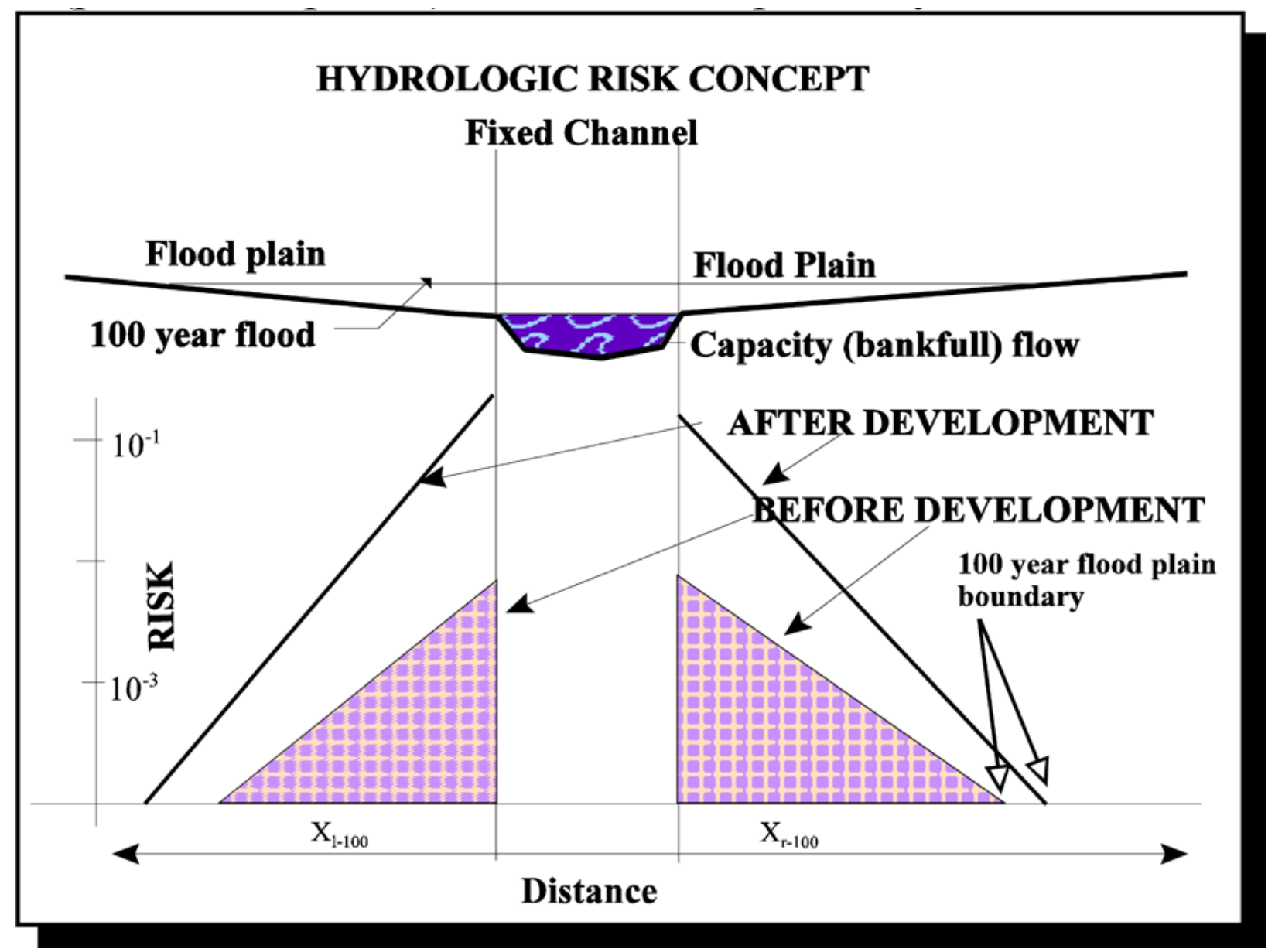

15 Novotny, Clark, Griffin, and Booth 
Figure 3

$R=\int_{0}^{\infty} r_{l}(x) d x+\int_{0}^{+\infty} r_{\gamma}(x) d x=r(0) \int_{0}^{\infty}\left[10^{-X_{1} x_{1}}+10^{-X_{*} x_{*}}\right] d x$

16 Novotny, Clark, Griffin, and Booth 
Figure 4: Concept of Risk Calculation for an Individual Stressor

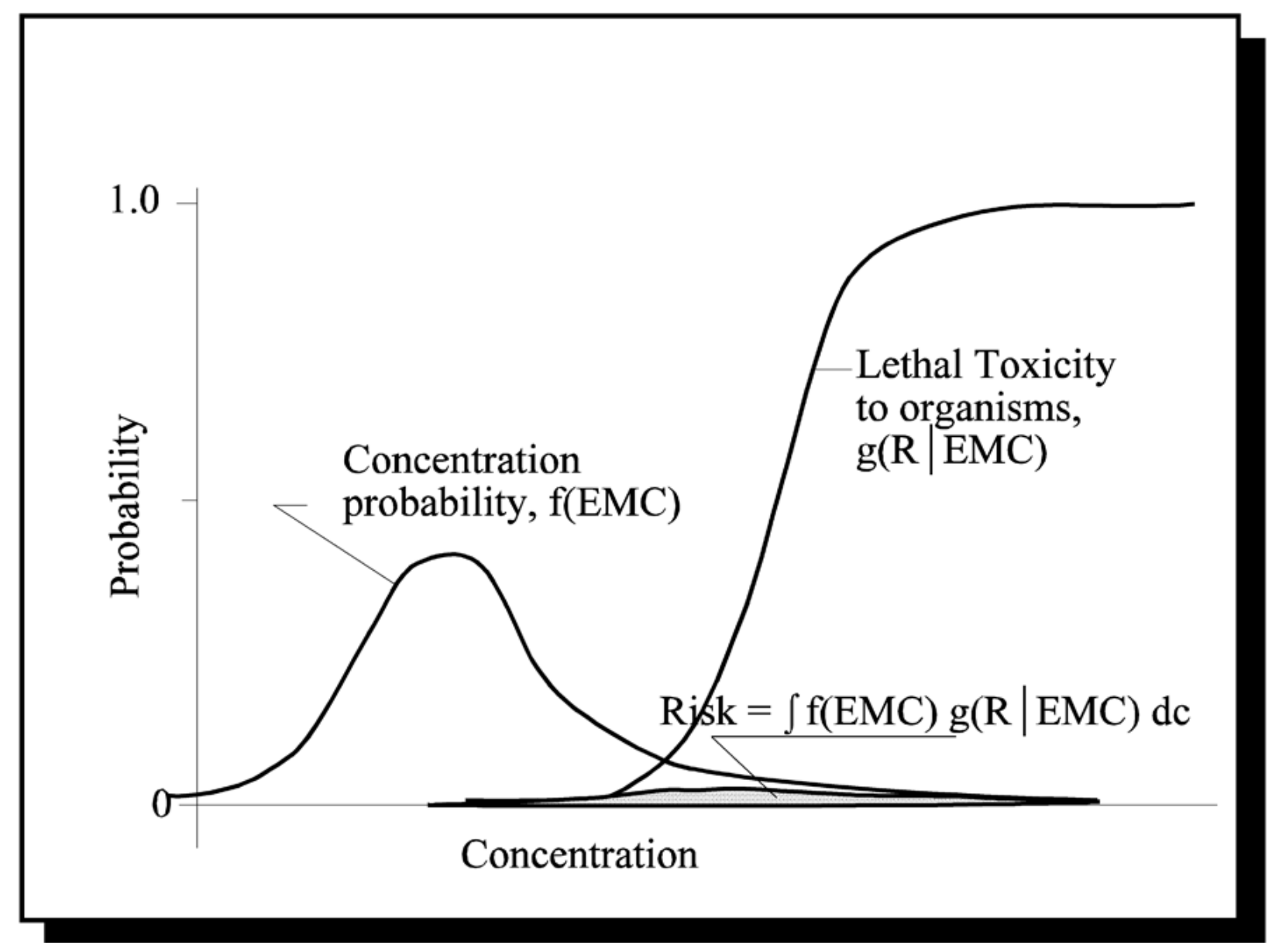

17 Novotny, Clark, Griffin, and Booth 